\title{
Rehydration of Digalactosyldiacylglycerol Model Membrane Lyophilizates Observed by NMR and Sorption Isotherm
}

\author{
H. HarańczyK ${ }^{a, *}$, M. Bacior ${ }^{a}$, J. Jamróz ${ }^{a}$, M. JemioŁa-Rzemińska ${ }^{b}$, And K. StrzaŁka ${ }^{b}$ \\ ${ }^{a}$ Institute of Physics, Jagiellonian University \\ Reymonta 4, 30-059 Cracow, Poland \\ ${ }^{b}$ Faculty of Biochemistry, Biophysics and Biotechnology, Jagiellonian University \\ Gronostajowa 7, 30-387 Cracow, Poland
}

\begin{abstract}
The initial stages of rehydration of digalactosyldiacylglycerol model membrane lyophilizates were observed using hydration kinetics, sorption isotherm, and high power proton relaxometry (at $30 \mathrm{MHz}$ ). Hydration time courses are single exponential and the sorption isotherm is sigmoidal in form. The mass of water saturating primary binding sites equals $\Delta M / m_{0}=0.019 \pm 0.001$. Proton free induction decays distinguish (i) immobilized protons of solid matrix of lyophilizate, signal $S_{0}$; (ii) protons of water fraction tightly bound to the lyophilizate surface, $L_{1}$; and (iii) mobile protons of loosely bound water pool, $L_{2}$. Hydration dependence of total water signal $\left(L_{1}+L_{2}\right) / S_{0}$ shows the presence of water fraction "sealed" in liposome structures, which equals $\Delta M_{\mathrm{S}} / m_{0}=0.092 \pm 0.007$.
\end{abstract}

PACS numbers: $82.56 . \mathrm{Na}$

\section{Introduction}

It is well known that drought conditions sorely decrease crop production. Therefore, a great effort is made to investigate the effects of water deficit stress on plant growth and survival. In some cases repeated stress treatments or slowly progressing stress led to an acclimation to dehydration. Among plants there exist species tolerant only to a mild water deficit, and also organisms resistant to deep desiccation (and frost), e.g. resurrection plants $[1,2]$, lichens ([3] and references therein).

At the cellular level dehydration results in implications for the photosynthetic membranes, where the light reaction of photosynthesis occurs. Therefore, the efficient recovery of photosynthetic membranes from water deficit stress to the native form is of great importance for plant growth and productivity. Photosynthetic membrane lyophilizates recover to lamellar phase after rehydration with a significant contribution of small membrane fragments from the disrupted lamellae $[4,5]$. However, it has not been clear yet whether photosynthetic membrane lyophilizates retain lamellar phase.

Among the four glycerolipids, digalactosyldiacylglycerol (DGDG) is the second abundant and it accounts for about $30 \%$ of the total lipids present in the thylakoid membranes of the chloroplasts. Along with monogalactosyldiacylglycerol (MGDG), DGDG has not only unique headgroup but it is also unusual in containing

* corresponding author; e-mail: hubert.haranczyk@uj.edu.pl two highly unsaturated fatty acid chains (linoleic and linolenic acid). The exceptional chemical structures of MGDG and DGDG give them unusual packing properties. The headgroup of DGDG is larger than that of MGDG and as the consequence DGDG molecule has a cylindrical shape and adopts a bilayer structure in contrast to MGDG which shows a tendency to pack into so-called hexagonal $\mathrm{H}_{\mathrm{II}}$ structure. In native membranes, DGDG binds at the periphery of the light harvesting complex $\left(\mathrm{LHC}_{\mathrm{II}}\right)$ trimer and allows the trimers to pack into two-dimensional crystalline arrays. Thus, this lipid is thought to be involved in lipid-mediated contacts between adjacent trimers.

We studied rehydration of lyophilized liposomes, composed of DGDG, measuring the number and distribution of water binding sites, sequence and kinetics of their saturation, as well as formation of tightly and loosely bound water pools at different steps of rehydration process. Taking advantage of NMR experiments, we examined the presence of water fraction "sealed" in liposome structure and compared its amount with the "sealed" water fraction in lyophilizates of developing and mature wheat photosynthetic membranes [6].

\section{Materials and methods}

The appropriate amounts of DGDG (Lipid Products, England) dissolved in chloroform were evaporated under gaseous nitrogen to form a thin film on the glass test tube walls. To remove the remnants of the solvent, the samples were subsequently exposed to vacuum for $1 \mathrm{~h}$. 
Dry lipid films were suspended in $5 \mathrm{ml}$ of distilled water and the multilamellar liposomes (MLV) were prepared by vortexing the samples at the room temperature for 15 min, until the optically uniform white opaque mixture was obtained. Then the solution of DGDG liposomes was frozen in liquid nitrogen temperature and lyophilized overnight.

The obtained DGDG lyophilizates in air-dry form were hydrated to $\Delta m / m_{0}=0.021 \pm 0.003$, where $m_{0}$ is the dry mass of the sample, and $\Delta m$ is mass of water taken up by liposome (determined gravimetrically). Before the hydration experiments the samples were incubated for 50 or $100 \mathrm{~h}$ over silica gel (at relative humidity, $p / p_{0}=$ $0 \%$ ), dehydrating to the hydration level $\Delta m / m_{0}=$ $0.012 \pm 0.005$.

The hydration courses were performed from the gaseous phase with the controlled humidity, at room temperature, over the surface of $\mathrm{H}_{3} \mathrm{PO}_{4}\left(p / p_{0}=9 \%\right)$, over the surfaces of saturated solutions of $\mathrm{KC}_{2} \mathrm{H}_{3} \mathrm{O}_{2}\left(p / p_{0}=\right.$ $23 \%), \mathrm{CaCl}_{2}\left(p / p_{0}=32 \%\right), \mathrm{K}_{2} \mathrm{CO}_{3}(44 \%), \mathrm{Na}_{2} \mathrm{Cr}_{2} \mathrm{O}_{7}$ $\left(p / p_{0}=52 \%\right), \mathrm{NH}_{4} \mathrm{NO}_{3}(63 \%), \mathrm{Na}_{2} \mathrm{~S}_{2} \mathrm{O}_{3}(76 \%), \mathrm{K}_{2} \mathrm{CrO}_{3}$ $(88 \%), \mathrm{Na}_{2} \mathrm{SO}_{4}(93 \%), \mathrm{K}_{2} \mathrm{SO}_{4}(97 \%)$, and over a water surface $\left(p / p_{0}=100 \%\right)$.

After completing the hydration courses, the dry mass of the samples was determined after heating at $70^{\circ} \mathrm{C}$. After duration of heating equal to $72 \mathrm{~h}$, as suggested by Gaff [7] measurable decrease in mass was still recorded. The minimal heating time for proper dehydration of the DGDG lyophilized liposomes to dry mass was in our experiments equal to $120 \mathrm{~h}$.

Proton free induction decays (FIDs) were measured on WNS HB-65 high power relaxometer (Waterloo NMR Spectrometers, St. Agatha, Ontario, Canada). The resonance frequency was $30 \mathrm{MHz}$ (at $B_{0}=0.7 \mathrm{~T}$ ); the transmitter power was $400 \mathrm{~W}$; the pulse length $\pi / 2=1.5 \mu \mathrm{s}$. Data were acquired using Compuscope 2000 card in an IBM clone computer, controling the spectrometer, and averaged over 2000 accumulations. Repetition time was $2.003 \mathrm{~s}$. The measurements were performed at room temperature.

The data were analyzed using the one-dimensional, FID analyzing procedure of the two-dimensional (in time domain) NMR signal-analyzing program CracSpin written at the Jagiellonian University, Cracow [8].

\section{Results}

The hydration courses for DGDG lyophilizates performed from the gaseous phase at the full range of target humidities, $p / p_{0}$, controlled between $9 \%$ and $100 \%$, were fitted well by single exponential function (see Fig. 1)

$$
\Delta m(t) / m_{0}=A_{0}+A_{1}\left[1-\exp \left(-t / t^{\mathrm{h}}\right)\right],
$$

where $\Delta m / m_{0}$ is the relative mass increase, $A_{0}$ is the saturation level for very tightly bound water fraction level, $A_{1}$ is the saturation level for the fast component, and $t^{\mathrm{h}}$ is the corresponding hydration time constant. The very tightly bound water fraction saturates for the hydration times shorter than the first measuring point (10 min).

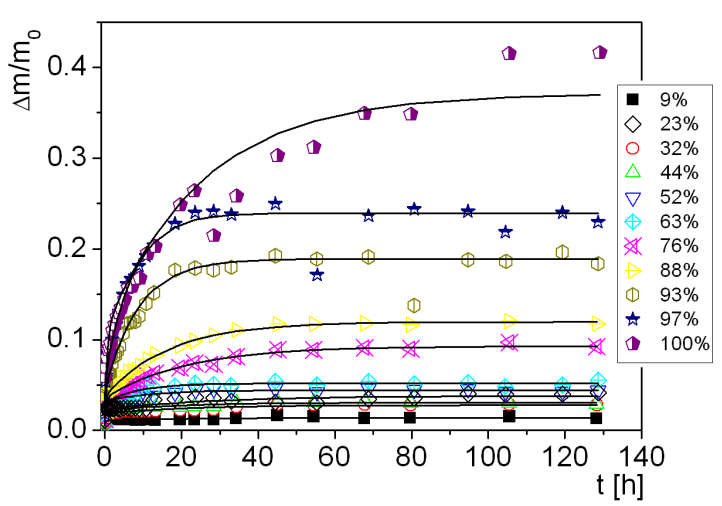

Fig. 1. Hydration kinetics of DGDG lyophilizate performed from the gaseous phase (the target humidities are marked in the inset). The errors are within the plot symbols.

The averaged over the all target humidities, $A_{0}$, equals $0.021 \pm 0.005$, whereas the hydration constant equals $t^{\mathrm{h}}=$ $(7.6 \pm 2.8) \mathrm{h}$.

The fluctuation in mass of water taken up during the hydration course performed at $p / p_{0}=100 \%$ may be caused either by fine structural changes of water in hydrated DGDG sample or by day-night slight temperature fluctuations in the lab. We fitted single exponential dependence, to get the averaged value of saturation hydration level. The saturation hydration level, $C^{\mathrm{h}}=A_{0}+A_{1}$, was taken for the construction of sorption isotherm.

For DGDG lyophilizates the sorption isotherm reveals sigmoidal form (Fig. 2), which usually is fitted by the Dent $[9,10]$ and/or the BET [11] model. Both models distinguish two types of water binding sites on the surfaces of the system investigated, namely: (i) "primary" water binding sites (directly to the adsorbent surface); and (ii) "secondary", usually weaker, water binding sites (to the surface binding sites with small affinity, to the primary bound water molecules, or to the previous water layers). The difference is that the BET model takes a fixed value of the ratio of the number of binding sites covered by $n$ water molecules to that covered by $n-1$ water molecules, $b=S_{n} /\left.S_{n-1}\right|_{h=1}=1$ (which is an artificial assumption), whereas in the Dent model this ratio may be varied between 0 and 1 (better modeling droplet formation).

The sorption isotherm for both models is described by

$$
C^{\mathrm{h}}(h)=\frac{\Delta M}{m_{0}} \frac{b_{1} h}{(1-b h)\left(1+b_{1} h-b h\right)},
$$

where $h$ is relative humidity $p / p_{0}$, expressed in absolute units, $\Delta M / m_{0}$ is the mass fraction of water saturating primary binding sites. At $h=1$ the contribution of empty primary binding sites, $S_{0}$, is expressed through the reciprocal of $b_{1}$ as $S_{0} /\left.S_{1}\right|_{h=1}=1 / b_{1}$.

To test the relevance of sorption model the sorption isotherm is usually presented in parabolic form (see Fig. 3). The parabolic form of the Dent isotherm is described by 


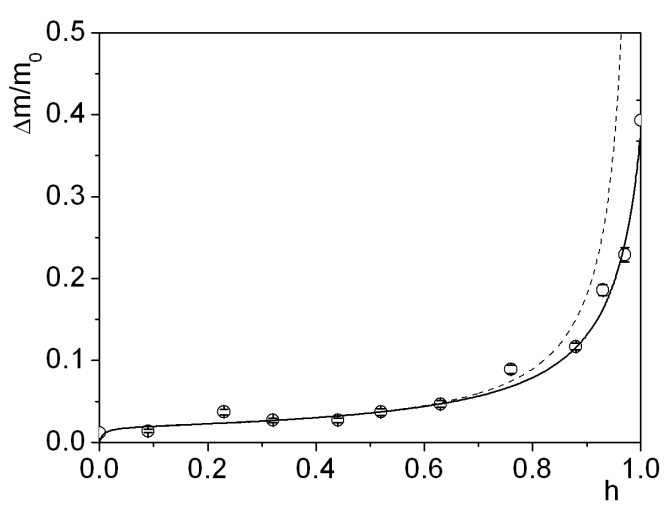

Fig. 2. The sorption isotherm for DGDG lyophilizates (solid line - Dent model, dashed line - BET model). The values of $h\left(=p / p_{0}\right)$ represent the relative humidity and the values of relative mass increase, $\Delta m / m_{0}$, are taken as the saturation values $C^{\mathrm{h}}$ from Eq. (2).

$$
\frac{h}{\Delta m / m_{0}}=A+B h-C h^{2},
$$

where parameters $\frac{\Delta M}{m_{0}}, b, b_{1}$ are connected with $A, B, C$ by the formulae

$$
\begin{aligned}
& b=\frac{\sqrt{B^{2}+4 A C}-B}{2 A}, \quad b_{1}=\frac{B}{A}+2 b, \\
& \frac{\Delta M}{m_{0}}=\frac{1}{A b_{1}} .
\end{aligned}
$$

The parabolic form of the BET isotherm is described by

$$
\frac{h}{\Delta m / m_{0}}=A+B h-(A+B) h^{2} .
$$

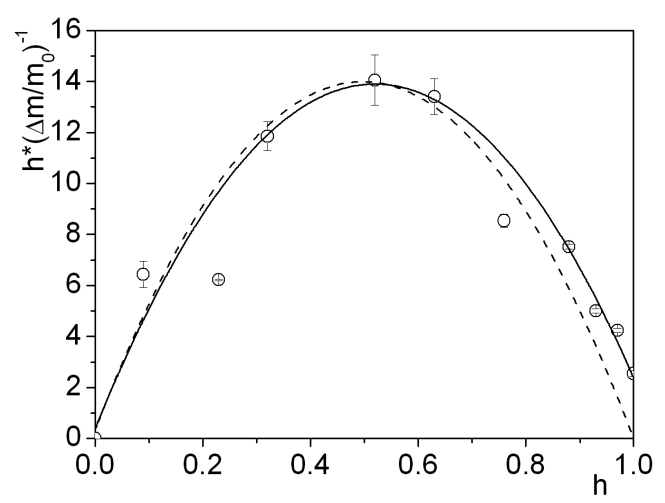

Fig. 3. Parabolic form of Dent and BET model (open circles - experimental data, solid line — fitted Dent model, dotted line - BET model).

For DGDG liposome lyophilizates the sorption isotherm is much better described by the Dent model (see Fig. 3). The mass of water saturating primary water binding sites $\Delta M / m_{0}=0.019 \pm 0.001$, which is comparable to photosynthetic membranes, in which DGDG is one of the main lipid constituents. For the native photosynthetic membranes $\Delta M / m_{0}=0.024$, whereas for ethylenediamine tetraacetic acid (EDTA) washed ones $\Delta M / m_{0}=0.017[6]$. The contribution from empty binding sites at $h=1$ is given by $1 / b_{1}=(0.7 \pm 0.24) \%$ (expressed as percentage), which is much less than that for native membranes $\left(1 / b_{1}=2.2 \%\right)$ and for EDTA washed $\left(1 / b_{1}=14.6 \%\right)$, reflecting the elevated hydrophilicity of DGDG (two hydrophilic galactose groups forming polar head) [6]. For DGDG lyophilized liposomes the model parameter $b$, indicating the applicability of the Dent model, is equal to $0.950 \pm 0.004$, a value slightly higher than that for photosynthetic membranes, e.g. for native membranes $b=0.896$, and for EDTA-washed $b=0.929$ [6].

The free induction decays for protons of the lyophilized DGDG liposomes, at lower hydration levels, are well fitted by the superposition of one Gaussian component, with the amplitude $S$, coming from the immobilized protons, and two exponential components, $L_{1}$ and $L_{2}$, coming from water tightly and loosely bound on the surfaces of liposome, whereas for higher hydration levels solely two exponential signal components are detected

$$
\begin{aligned}
& \mathrm{FID}(t)=S \exp \left(-\left(\frac{t}{T_{2 S}^{*}}\right)^{2}\right)+L_{1} \exp \left(-\frac{t}{T_{2 L_{1}}^{*}}\right) \\
& +L_{2} \exp \left(-\frac{t}{T_{2 L_{2}}^{*}}\right),
\end{aligned}
$$

where $T_{2 S}^{*}$ is the proton spin-spin relaxation time of solid component taken as the 1/e-value of Gaussian solid signal, and $T_{2 L_{1}}^{*}$ and $T_{2 L_{2}}^{*}$ are the relaxation times of proton liquid fractions $L_{1}$ and $L_{2}$, respectively. A typical FID is shown in Fig. 4.

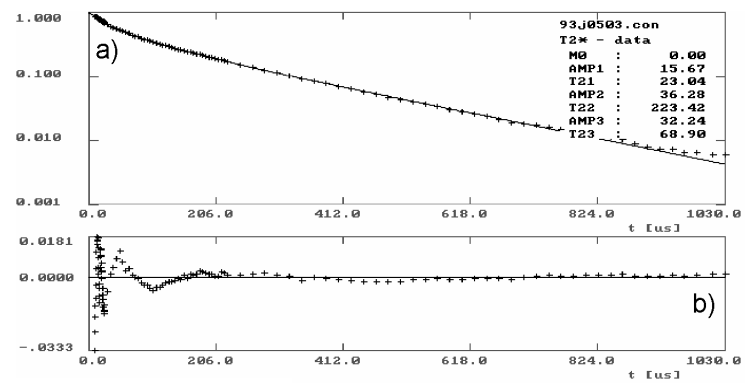

Fig. 4. (a) Proton free induction decay recorded for DGDG lyophilizates at $30 \mathrm{MHz}$; the pulse length $\pi / 2=$ $1.4 \mu \mathrm{s}$. The relative mass increase was $\Delta m / m_{0}=0.039$. $Y$-axis shows the normalized signal amplitude. The solid line represents a least squares fit of Eq. (6) to the data. (b) The residual function calculated as the difference between the fitted and recorded values of the FID signal, which for any recorded point does not exceed $3.3 \%$.

Figure 5 shows the rehydration dependence of the proton relaxation times for the components of FID signal of DGDG liposome lyophilizates. The solid signal was nearly Gaussian in form, the "beat" pattern was hardly seen even in residual function [12]. The spin-spin relaxation time for solid component was equal to $T_{2 S}^{*} \approx 20 \mu \mathrm{s}$, which is close to the value for the solid matrices of several 
dry microheterogeneous biological systems (e.g. photosynthetic membranes [6], bark and bast [13], wheat seed [14], lichen thallus [15-18], dentine and dental enamel [19]). Thus, we assigned the Gaussian component, $S_{0}$, to solid matrix of the wheat thylakoid lyophilizate, and used this amplitude as a unit to scale the amplitudes of the other signal components.

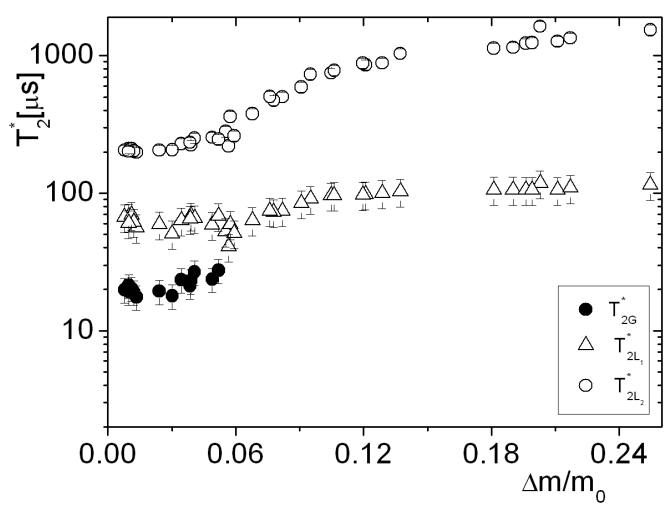

Fig. 5. The hydration dependence of proton FID relaxation times for DGDG lyophilizates. Solid Gaussian, $S$, component - closed circles, tightly bound water, $L_{1}$, component - open triangles, and loosely bound water, $L_{2}$, component - open circles.

For the mobile proton fraction $L_{1}$, the value of the decay time does not depend much on the hydration level and equals $T_{2 L_{1}}^{*} \approx 90 \mu \mathrm{s}$. Proton FID time for the $L_{1}$ fraction resembles that for the tightly bound water signal of photosynthetic membranes [6], bark and bast [13], wheat seed [14], lichen thallus [15-18], dentine and dental enamel [19], and of the control porous glass [20].

The relaxation time $T_{2 L_{2}}^{*}$ for longest mobile signal component increases with the increasing hydration level (Fig. 5), suggesting that this component may be an average of some proton subsystems being in fast exchange regime [21]. For higher hydration levels the $T_{2 L_{2}}^{*}$ values are of the order of $\approx 1 \mathrm{~ms}$. Thus, the $L_{2}$ component is a loosely bound water fraction, or free water. The spinspin relaxation times $T_{2 L_{2}}^{*}$ measured in FID experiment are shortened by $B_{0}$ inhomogeneities [22]:

$$
\frac{1}{T_{2}^{*}}=\frac{1}{T_{2}}+\frac{\gamma \Delta B_{0}}{2}
$$

where $T_{2}$ is spin-spin relaxation time, $\gamma$ is gyromagnetic ratio, and $\Delta B_{0}$ is a change of magnetic field $B_{0}$ within the sample. Although the solid and short exponential components in the FID experiment are not changed by $\Delta B_{0}$ as compared to those measured by the Carr-PurcellMeiboom-Gill (CPMG) echo train, the measured $T_{2 L_{2}}^{*}$ are significantly shortened.

Proton FID recorded from dehydrated DGDG membrane lyophilizate reveals measurable content of mobile component, which calculated from the hydration dependence of the total liquid signal (Fig. 6), equals $\left(L_{1}+L_{2}\right) / S=3.165 \pm 0.149$. This parameter expressed in units of relative mass increase gives the value

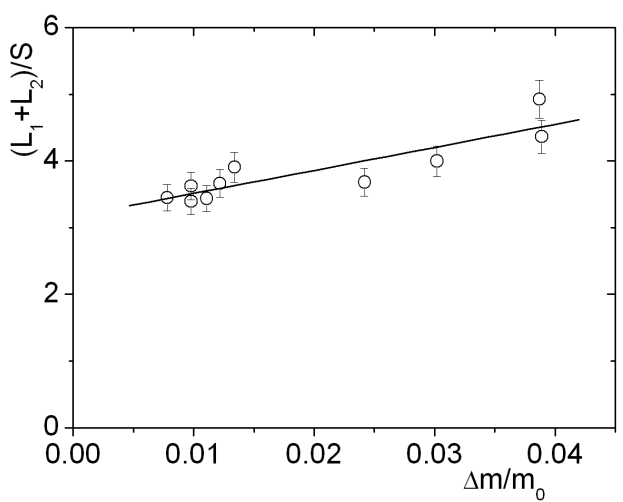

Fig. 6. The $\left(L_{1}+L_{2}\right) / S$ hydration dependence for rehydrated DGDG membrane lyophilizates. The solid line was calculated from $\left(L_{1}+L_{2}\right) / S=(34.541 \pm$ $6.540)\left(\Delta m / m_{0}\right)+(3.165 \pm 0.149)$.

$\Delta m / m_{0}=0.092 \pm 0.007$. The contribution of tightly bound water fraction in dehydrated DGDG membrane lyophilizate is $L_{1} / S=1.304 \pm 0.085$, which equals $\Delta m_{1} / m_{0}=0.065 \pm 0.008$.

\section{Discussion}

The mass of very tightly bound water fraction calculated from hydration kinetics is $A_{0}=0.021 \pm 0.005$, which corresponds to $1.10 \pm 0.26$ water molecule per $1 \mathrm{DGDG}$ molecule, whereas water fraction saturating the primary water binding sites, $\Delta M / m_{0}=0.019 \pm 0.001$, which is $0.99 \pm 0.05$ water molecule per 1 DGDG molecule. Most likely this is the same water fraction, and we may assume that it is one water molecule per one lipid.

Proton NMR data revealed the presence of significant fraction of water, which is retained in dry lyophilizate. Its mass equals $\Delta m / m_{0}=0.092 \pm 0.007$, which is $4.80 \pm 0.37$ water molecules/DGDG molecule. The tightly bound water contribution makes up $\Delta m_{1} / m_{0}=0.065 \pm 0.007$, which is $3.39 \pm 0.37$ water molecules per DGDG molecule. It contains both tightly bound water fraction signal, $L_{1}$, and loosely bound water signal, $L_{2}$, which means that it is not differentiated by the proximity to membrane surface, but it consists of water "sealed" in DGDG membrane structures. Since the content of "sealed" water fraction is higher than that in photosynthetic membrane lyophilizates (where $\Delta m / m_{0}=0.055[6]$ ), it may suggest that this fraction of water molecules localizes in lipid regions of photosynthetic membrane lyophilizate. We may consider that they are placed inside inverted hexagonal phase domains. The structure of this kind consists of rod-like aggregates which are formed by the polar lipid headgroups located at the center of the rods along with some water, while the fatty acid chains point outward so that the surfaces of the rod are hydrophobic. It is worth mentioning that the presence of $\mathrm{H}_{\mathrm{II}}$ phase, has been detected in the native photosynthetic membranes $[5,23]$ as well as in phosphatidylcholine liposomes containing MGDG at the amount ranging 50\% [24]. 
Moreover, since seeds are a plant material often used for investigations of the effects of dehydration, one may consider the Simon hypothesis. Based on studies on seeds during imbibition and first stages of germination it suggests that at water content lower than $20 \%$ a phase transition from La to $\mathrm{H}_{\mathrm{II}}$ occur in plant cell membranes [25]. However, Seewaldt et al. examining by X-ray crystallography and NMR measurements isolated soybean seed lipids as well as particles of seed tissues found no proof for a lipid phase change induced by the shortage of water [26]. There is now evidence supporting the mechanism where in vivo sucrose may substitute for water, enabling the complex lipids to retain the characteristics of hydrated lipids even under the water deficit.

In general, there is no doubt that dehydration and hydration of membranes have an impact on membrane properties. Along with chemical alteration of the lipid composition which are the subject of intensive studies, the factor that plays a crucial role for membrane structure and function is the physical behavior of the lipids. The freeze fracture micrograms show that water stress is a reason of cell dehydration and leads to a reorganization of membrane components ([27] and the references therein, which is reflected by the occurrence of the areas free of intramembranous particles, and well correlates with damage executed as ion leakage or no growth. There are also indications of phase transitions from $L_{\alpha}$ to $\mathrm{H}_{\mathrm{II}}$. Furthermore, electron microscopic data reveal the presence of bicontinuous cubic phase probably as an intermediate to reverse hexagonal phase. However, the consequences of water deficit stress observed in case of plants submitted to an acclimation process may differ from that of the non- acclimated ones, implying a larger resistance and higher possibility of survival.

\section{References}

[1] F. Navarizzo, F. Ricci, C. Vazzana, M.F. Quartacci, Physiol. Plant. 94, 135 (1995).

[2] K. Schneider, B. Wells, E. Schmelzer, F. Salamini, D. Bartels, Planta 189, 120 (1993).

[3] H. Harańczyk, On Water in Extremely Dry Biological Systems, Wydawnictwo Uniwersytetu Jagiellońskiego, Kraków 2003.

[4] H. Harańczyk, K. Strzałka, T. Bayerl, G. Klose, J.S. Blicharski, Photosynthetica 19, 414 (1985).

[5] H. Harańczyk, K. Strzałka, W. Dietrich, J.S. Blicharski, J. Biol. Phys. 21, 125 (1995).
[6] H. Harańczyk, A. Leja, K. Strzałka, Acta Phys. Pol. A 109, 389 (2006)

[7] D.F. Gaff, Oecologia (Berl.) 31, 95 (1977).

[8] W. Wegglarz, H. Harańczyk, J. Phys. D, Appl. Phys. 33, 1909 (2000).

[9] R.W. Dent, Textile Res. J. 47, 145 (1977).

[10] A.J. Hailwood, S. Horrobin, Trans. Faraday Soc. B 42, 84 (1946).

[11] S. Brunauer, P.H. Emmett, E. Teller, J. Am. Chem. Soc. 60, 309 (1938).

[12] W. Derbyshire, M. Van Den Bosch, D. Van Dusschoten, W. MacNaughtan, I.A. Farhat, M.A. Hemminga, J.R. Mitchell, J. Magn. Res. 168, 278 (2004).

[13] H. Harańczyk, W.P. Wȩglarz, S. Sojka, Holzforschung 53, 299 (1999).

[14] H. Harańczyk, K. Strzałka, G. Jasiński, K. Mosna-Bojarska, Coll. Surf. A 115, 47 (1996).

[15] H. Harańczyk, S. Gaździński, M.A. Olech, New Phytologist 138, 191 (1998).

[16] H. Harańczyk, S. Gaździński, M. Olech, New Aspects in Cryptogamic Research, Contribution in Honour of Ludger Kappen. Bibl. Lichenol. 75, 265 (2000).

[17] H. Harańczyk, S. Gaździński, M. Olech, Mol. Phys. Rep. 29, 135 (2000).

[18] H. Harańczyk, A. Pietrzyk, A. Leja, M.A. Olech, Acta Phys. Pol. A 109, 411 (2006).

[19] N. Funduk, G. Lahajnar, L. Miljković, S. Skočajić, D.W. Kydon, L.J. Schreiner, M.M. Pintar, Zobozdrav. Vestn. 41, (Suppl. 1), 139 (1986).

[20] H. Harańczyk, K.G. Soga, R.J. Rumm, M.M. Pintar, Magn. Res. Imag. 9, 723 (1991).

[21] J.R. Zimmerman, W.E. Brittin, J. Phys. Chem. 61, 1328 (1957)

[22] A. Timur, J. Petroleum Technol. 21, 775 (1969).

[23] K. Gounaris, A. Sen, A.P.R. Brain, P.J. Quinn, W.P. Williams, Biochim. Biophys. Acta 728, 129 (1983).

[24] D. Latowski, J. Kruk, K. Burda, M. Skrzynecka-Jaskier, A. Kostecka-Gugała, K. Strzałka, Eur. J. Biochem. 269, 4656 (2002).

[25] E.W. Simon, in: Dry Biological Systems, Eds. J.H. Crowe, J.S. Clegg, Academic Press, London 1978, p. 205.

[26] V. Sewaldt, D.A. Priestley, A.C. Leopold, G.W. Feigenson, F. Goodsaid-Zalduondo, Planta 152, 19 (1981).

[27] C.S. Liljenberg, Prog. Lipid. Res. 31, 335 (1992). 